\title{
Dynamic MR Imaging of the Temporomandibular Joint Using a Balanced Steady-State Free Precession Sequence at 3T
}

\author{
P. Yen, R.W. Katzberg, M.H. Buonocore, and J. Sonico
}

\begin{abstract}
SUMMARY: The aim of our project was to develop an MR imaging protocol for dynamic imaging of the TMJ. We imaged a total of 24 joints in 12 subjects. We developed an imaging protocol on a 3T system using the true FISP sequence that yielded an acceptable spatial and temporal resolution for dynamic MR imaging.
\end{abstract}

ABBREVIATIONS bSSFP = balanced steady-state free precession; FISP = fast imaging with steady-state precession; HASTE = half-Fourier acquired single-shot turbo spin-echo; TMJ = temporomandibular joint

$\mathrm{T}$

he TMJ is one of the most complex joints to image, and there

has only been limited success in performing true dynamic MR imaging. The articular disk is a biconcave fibrous structure located between the surface of the condyle inferiorly and the articular eminence superiorly. ${ }^{1}$ Internal derangement is defined as an abnormal anatomic relationship between the disk and the mandibular condyle.

Functional and dysfunctional aspects of disk physiology include the following: 1) coordinated (normal) disk function; 2) disk displacement with reduction; and 3) disk displacement without reduction. ${ }^{2}$ If the disk is abnormally displaced anterior to the bony condyle yet snaps or clicks into a normal anatomic position when the jaw opens, the terminology for this condition is "disk displacement with reduction." Clinically, this occurs with both opening and closing clicking sounds and is often associated with joint, muscular, and facial pain. Disk displacement with reduction is thus both an anatomic and a functional disorder and should be classified as early, intermediate, or late reduction of the disk, depending on the distance from the joint at which the disk re-assumes its normal anatomic relationship as the bony condyle translates forward with jaw opening. This general classification along with the anatomic location of the reciprocal displacement on jaw closing serves to stage the severity of the disk displacement

Received April 23, 2011; accepted May 11.

From the Department of Radiology, University of California Davis Medical Center, Sacramento, California.

Please address correspondence to Philip Yen, MD, Department of Radiology, University of California Davis Medical Center, 4860 Y St, Suite 3100, Sacramento, CA 95817; e-mail: philipyenmd@gmail.com

Indicates article with supplemental on-line video.

http://dx.doi.org/10.3174/ajnr.A2734 and is useful in determining whether protrusive splint therapy is likely to be successful. ${ }^{2}$

An additional value of functional imaging is the ability to reliably distinguish clicking sounds that are clinically similar to disk displacement with reduction. These commonly include the clicking or popping sounds that are associated with interference of the articular eminence ("eminence clicks") of the base of the skull when the condyle and disk move underneath and anteriorly beyond that structure. Structural deformities of the disk itself or condyle can also produce clicking sounds with jaw opening and closing, regardless of whether the disk is displaced.

The functional dynamics of TMJ internal derangement were optimally depicted by the invasive arthrographic technique in the late 1970s to late 1980s. This technique was difficult to perform and often painful to the patient. With the advent of noninvasive imaging techniques such as CT and MR imaging, the arthrographic technique has been generally abandoned. However, the obvious limitation of the noninvasive techniques of CT and MR imaging is the lack of dynamic imaging. CT has also been abandoned due to relatively poor anatomic resolution and the high radiation dose to the lens of the eye. Currently, the most popular imaging technique is MR imaging. ${ }^{3,4}$ However, the ability to distinguish the various etiologies of TMJ joint sounds or to classify the clinical degree of disk displacement with reduction is not satisfactory. Technologic advances in MR imaging, especially the relatively recent availability of ultrashort TR imaging with bSSFP magnetization dynamics and use of higher field strengths, offer the potential to re-establish the dynamic imaging component in the clinical evaluation that was lost with the abandonment of arthrography.

The aim of our study was the development of an MR imaging protocol that enables dynamic MR imaging of the TMJ. 

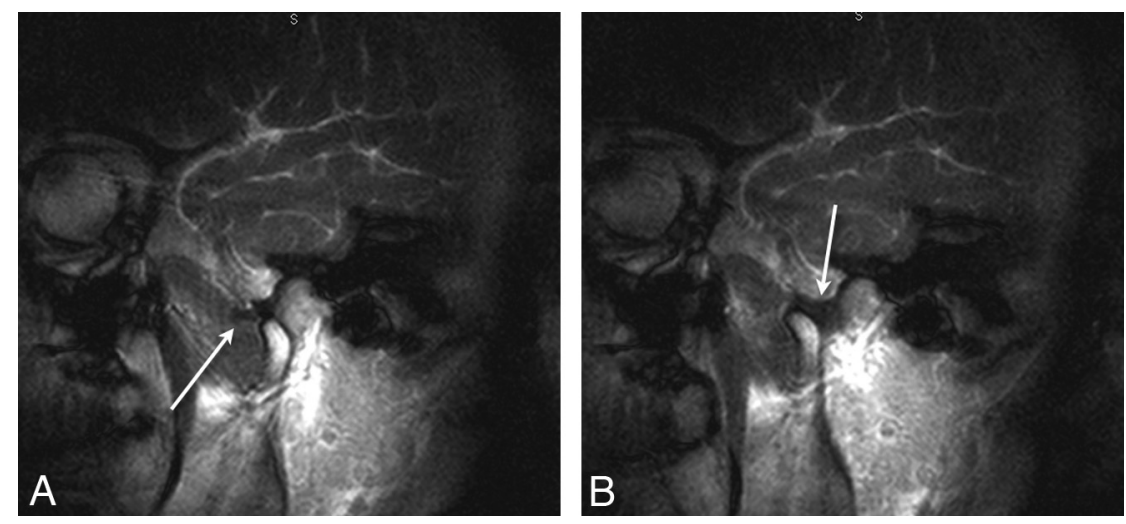

FIG 1. A, A 28-year-old man with a unilateral left opening and closing joint clicking. Images acquired by using true FISP with a 3.6-ms TR, 1.8-ms TE, $150 \times 150 \mathrm{~mm}$ FOV, $128 \times 128$ raw data matrix, and a 6-mm section thickness. A single frame obtained from dynamic sagittal images in a corrected plane determined from the dynamic axial images shows an anteriorly displaced disk (arrow) just before recapture. B, A single frame from the same true FISP imaging series shows reduction of the displaced disk (arrow), which is clinically manifest as the opening click. The On-line Video includes the entire dynamic series.

\section{TECHNIQUE}

Our study was approved by the institutional review board of our institution. After informed consent, 12 volunteer subjects (24 TMJs) with clinically normal joints, clicking joints, and longstanding TMJ pain and dysfunction were initially imaged during 1- to 1.5-hour sessions to establish optimal dynamic images. Subjects were enrolled on referral from dental clinicians made aware of the objectives of the study. Subsequent imaging sessions required only 30-45 minutes. This investigation was performed on a $3 \mathrm{~T}$ Trio MR imaging system (Siemens Healthcare, Erlangen, Germany) by using a bitemporal 4-channel array surface coil (Bitemporal Phased Array model NMSG003A, Nova Medical, Wilmington, Massachusetts).

The initial protocol included the acquisition of standard 3-mm-section-thickness fast spin-echo images of both TMJs, in static closed and opened jaw positions and with both protonattenuation and T2 weighting. It also included acquisition of T1weighted 3-mm-section-thickness coronal fast spin-echo images of the static closed jaw. In initial tests to determine optimal dynamic imaging, EPI, HASTE, and true FISP pulse sequences were evaluated. Axial and sagittal images were acquired separately during jaw motion by using 3-, 6-, and 9-mm section thicknesses, varying spatial and temporal resolution, and varying scanning times. The optimal dynamic images were chosen by consensus by coinvestigators, 1 of whom has $>30$ years' experience in clinical TMJ assessment, which included TMJ arthrographic dynamic imaging, having familiarity with fluoroscopically observed functional imaging and diagnosis.

The optimal imaging sequences were achieved with true FISP, which is classified in manufacturer independent terminology as a bSSFP pulse sequence, using a 3.6-ms TR, 1.8-ms TE, $150 \times 150$ $\mathrm{mm}$ FOV, 6-mm section thickness, and a $128 \times 128$ raw data matrix collected by using a $4 / 8$ partial Fourier acquisition. This acquisition yielded an effective voxel size of $1.2 \times 1.2 \times 6 \mathrm{~mm}^{3}$. The dynamic imaging scan consisted of continuous scanning for 16.5 seconds during slow opening and closing of the jaw (approximately 6 seconds per cycle of jaw opening and closing). Each image was acquired in $267 \mathrm{~ms}$ for a total of 62 images during continuous scanning. Acquisitions with smaller (eg, $96 \times 96$; better temporal, but poorer spatial resolution) and larger (eg, $160 \times 160$; better spatial, but poorer temporal resolution) raw data matrices were also evaluated in comparison with the initially tested $128 \times 128$ raw data matrix described above. The images acquired with $128 \times 128$ raw data matrix and 6-mm section thickness yielded the best dynamic evaluation of the TMJ.

Disk position and dynamics were best depicted in a sagittal plane of imaging that varied between subjects and often between joints in the same subject. The axial dynamic imaging plane was essential in imaging in the actual path of condylar and disk movement in the sagittal plane with opening and closing of the jaw.

Dynamic images were acquired successfully in all joints, and repetitive imaging was rapidly feasible. Determination of the locations of the opening disk recapture (opening click) and re-displacement of the disk with jaw closing was readily accomplished. Discrimination between eminence clicks and disk displacement with reduction was possible, but our sample size was much too small for a rigorous comparison.

\section{DISCUSSION}

Dynamic imaging of the TMJ has been performed with limited success, but prior attempts have been at $1.5 \mathrm{~T}$. Chen et $\mathrm{al}^{5} \mathrm{de}-$ scribed some success with dynamic MR imaging of the TMJ by using the EPI pulse sequence. Their visualization of the disk was limited due to relatively low SNR and spatial resolution. Wang et $\mathrm{al}^{6}$ visualized the disk adequately by using the HASTE pulse sequence, but their temporal resolution was limited to 1.17 seconds by using that sequence. Abolmaali et $\mathrm{al}^{7}$ and Shimazaki et al ${ }^{8}$ reported adequate visualization of the disk by using the true FISP sequence with a reported temporal resolution of $\leq 255 \mathrm{~ms}^{7}$

While we explored the EPI and HASTE sequences, the best visualization of the TMJ was obtained in images acquired with the true FISP sequence. Because the true FISP sequence creates a very large steady-state magnetization, it provides an intrinsically high SNR, and because it uses an ultrashort TR, very high spatial resolution can be obtained in reasonable scanning times. ${ }^{7,9}$ Use of a $3 \mathrm{~T}$ system provides increased thermal equilibrium magnetization, which further improves the SNR, allowing higher spatial resolution (by decreasing the FOV) or higher temporal resolution (by reducing the FOV and raw data matrix size). Our optimized parameters allowed acquisition of 62 images during slow jaw motion during a total scanning time of 16.5 seconds, corresponding to a temporal resolution of $267 \mathrm{~ms}$.

The disk and the normal mechanics of the TMJ could be visualized during the entire course of jaw opening and closing as well as the dynamics of internal derangement. For example, in a subject who reported an opening and closing click in 1 joint, we were able to identify and characterize disk displacement with subsequent reduction (Fig $1 A,-B$ and On-line Video). We were specif- 
ically able to identify the exact anatomic condylar position relative to the glenoid fossa and eminence at which the disk was reduced. In addition, the disk morphology just before reduction was also well-depicted. In a second subject with unilateral joint clicking, the reduction phase was not as well-depicted, but the reciprocal displacement phase was optimally visualized. In both cases, the location of the condyle from the fossa where the disk reduction occurred was readily ascertained. We also observed disk displacement without reduction in 2 TMJs, but the dynamic images did not appear to offer any additional information.

It has been shown that the disk can undergo rotational or sideways displacement, ${ }^{10}$ and Abolmaali et $\mathrm{al}^{7}$ reported $4 \mathrm{TMJs}$ that were not visualized due to lateral displacement. Our protocol was designed to detect any potential rotational or sideways displacement by imaging a single coronal plane and 3 separate sagittal planes through the medial, mid, and lateral aspects the TMJ. While we did not observe this form of internal derangement, we intend to further evaluate the efficacy of this protocol in detecting rotational or sideways displacement.

In conclusion, our protocol provides dynamic MR imaging of the TMJ for assessment of disk and condyle relationships. Specifically, the technique could be applied in clicking joints to rule out additional causes of joint noises including those caused by disk displacement with reduction. On $3 \mathrm{~T}$ MR imaging systems that have the bSSFP pulse sequence, this protocol is easily implemented and the required images can be acquired with only a few additional minutes of scanning time for each joint. We believe our protocol can be a valuable tool to supplement static MR imaging evaluation of the TMJ.
Disclosures: Richard W. Katzberg, UNRELATED: Grants/Grants Pending: Siemens.

\section{REFERENCES}

1. Rees LA. The structure and function of the mandibular joint. $\mathrm{Br}$ Dent K 1954;96:125-33

2. Katzberg RW. Temporomandibular joint imaging. Radiology 1989;170:297-307

3. Tomas X, Pomes J, Berenguer J, et al. MR imaging of temporomandibular joint dysfunction: a pictorial review. Radiographics 2006;26:765-81

4. Westesson PL, Katzberg RW, Tallents RH, et al. CT and MR of the temporomandibular joint: comparison with autopsy specimens. AJR Am J Roentgenol 1987;148:1165-71

5. Chen YJ, Gallo LM, Meier D, et al. Dynamic magnetic resonance imaging technique for the study of the temporomandibular joint. $J$ Orofac Pain 2000;14:65-73

6. Wang EY, Mulholland TP, Pramanik BK, et al. Dynamic sagittal halfFourier acquired single-shot turbo spin-echo MR imaging of the temporomandibular joint: initial experience and comparison with sagittal oblique proton-attenuation images. AJNR Am J Neuroradiol 2007;28:1126-32

7. Abolmaali ND, Schmitt J, Schwarz W, et al. Visualization of the articular disk of the temporomandibular joint in near-real-time MRI: feasibility study. Eur Radiol 2004;14:1889-94. Epub 2004 Jul 29

8. Shimazaki Y, Saito K, Matsukawa S, et al. Image quality using dynamic MR imaging of the temporomandibular joint with true-FISP sequence. Magn Reson Med Sci 2007;6:15-20

9. Fuchs F, Laub G, Othomo K. TrueFISP: technical considerations and cardiovascular applications. Eur J Radiol 2003;46:28-32

10. Katzberg RW, Westesson PL, Tallents RH, et al. Temporomandibular joint: MR assessment of rotational and sideways disk displacements. Radiology 1988;169:741-48 